\title{
Appetitive and Aversive Taste Conditioning in a Computer Game Influences Real-World Decision Making and Subsequent Activation in Insular Cortex
}

\begin{abstract}
Jonathan A. McCabe, ${ }^{1}$ Phillipe N. Tobler, ${ }^{2}$ Wolfram Schultz, ${ }^{2}$ Anthony Dickinson, ${ }^{3}$ Victoria Lupson, ${ }^{4}$ and Paul C. Fletcher ${ }^{1}$

Departments of ${ }^{1}$ Psychiatry, ${ }^{2}$ Physiology, Development and Neuroscience, and ${ }^{3}$ Psychology, and ${ }^{4}$ Wolson Braik vaging Centr University of Cambridge, Cambridge CB2 2QQ, United Kingdom

A stimulus, by virtue of its pairing with a rewarding or an aversive outcome, can a However, there is uncertainty concerning the extent to which such properties $n$ sought to determine whether conditioning-dependent motivational properties can to sfer from a computer game to the real world and, further, whether this conditioning might be expressed in terms of bros sonses is sured using functional magnetic resonance imaging ( $\mathrm{MMRI}$ ). We studied healthy participants conditioned with ave oive and appetitive drinks in the context of a virtual cycling race. Three days after conditioning, participants returned for a fMRI sess $\mathrm{n}$. We took his opportunity to observe the impact of incidental presentation of conditioned stimuli on a real-world decision (seat choic We foun a significant influence of conditioning on seat choice and, moreover, noted that individual susceptibility to this in was differential insula cortex responses during subsequent scanning. The choice was also predicted by participants erso scores and, as a statistical trend $(p=0.07)$, by their sense of immersion in the game environment. Our data show th motiva al properties of stimuli can transfer from the virtual to the real world. While much concern has been expressed over the i pact o virtua xperience on general levels of aggression and mood, our data point to another important consideration: the fact that a mulu vir al environment can acquire motivational properties that persist and modify behavior in the real world.
\end{abstract}

Key words: classical conditioning; moti th aversion, sward; insula; imaging

\section{Introduction}

Virtual environments areincreà gly use or both clinical and leisure purposes. The oson ion th ny people report when seeking rewards a overd ming obvacles within a computer game suggests that sones me mention of reality. It therefore becomes imp ant to understand whether experiences within a virtual setting ma transfer to real-world behavior. Direct evidence of such transfer would be informative to the current debate over the impact, harmful or beneficial, of increasing immersion in games and virtual environments.

Can experience in the virtual world alter behaviors in the real world? There is evidence that it can be used to improve motor skills in clinical training (Park et al., 2007). It can also provide an environment in which to optimize stroke treatment and recovery (Krakauer, 2006), to provide controlled

Received Aug. 19, 2008; revised Dec. 5, 2008; accepted Dec. 7, 2008

This work was performed within the Behavioral and Clinical Neurosciences Institute, jointly supported by the Medical Research Council and the Wellcome Trust. We thank the radiography team at the Wolfson Brain Imaging Centre for support in acquisition of the fMRI data.P.C.F. is supported by the Bernard Wolfe Health Neuroscience Fund and by the Wellcome Trust.

Correspondence should be addressed to Paul C. Fletcher, Department of Psychiatry, University of Cambridge, Box 189, Addenbrooke's Hospital, Hills Road, Cambridge CB2 2QQ, UK. E-mail: pcf22@cam.ac.uk.

DOl:10.1523/JNEUROSCI.3938-08.2009

Copyright $\odot 2009$ Society for Neuroscience $\quad 0270-6474 / 09 / 291046-06 \$ 15.00 / 0$ exposure therapy for treating fears of spiders (Carlin et al., 1997) and heights (Emmelkamp et al., 2002), and to resolve symptoms of posttraumatic stress disorder (Rothbaum et al., 2001; Pull, 2008). The latter, predominantly clinical, studies, suggest that virtual experience has some overlap with realworld experience but as yet there is no evidence of transfer of basic conditioned associations from one world to the other. Indeed, since the context in which a conditioned stimulus (CS) occurs may play an important role in the expression of the CS-US (unconditioned stimulus) association (Holland, 1985; Bouton and Swartzentruber, 1986), there may be reasons to suspect that a CS will not always directly transfer its effects between environments. Even a well-learned CS-US relationship may not be expressed when the context is changed.

In the current study (see Fig. 1), we investigated a pavlovian relationship involving simple conditioned food preference and aversions. We studied healthy humans to establish whether such conditioning within a virtual environment would have a measurable impact, several days later, upon realworld behavior and on brain responses to stimuli previously experienced in the virtual environment. To do this, we conditioned two novel, visual stimuli by presenting them in the context of a virtual cycling race (the stimuli appeared as logos on the jerseys of competitors). One stimulus became a pavlov- 

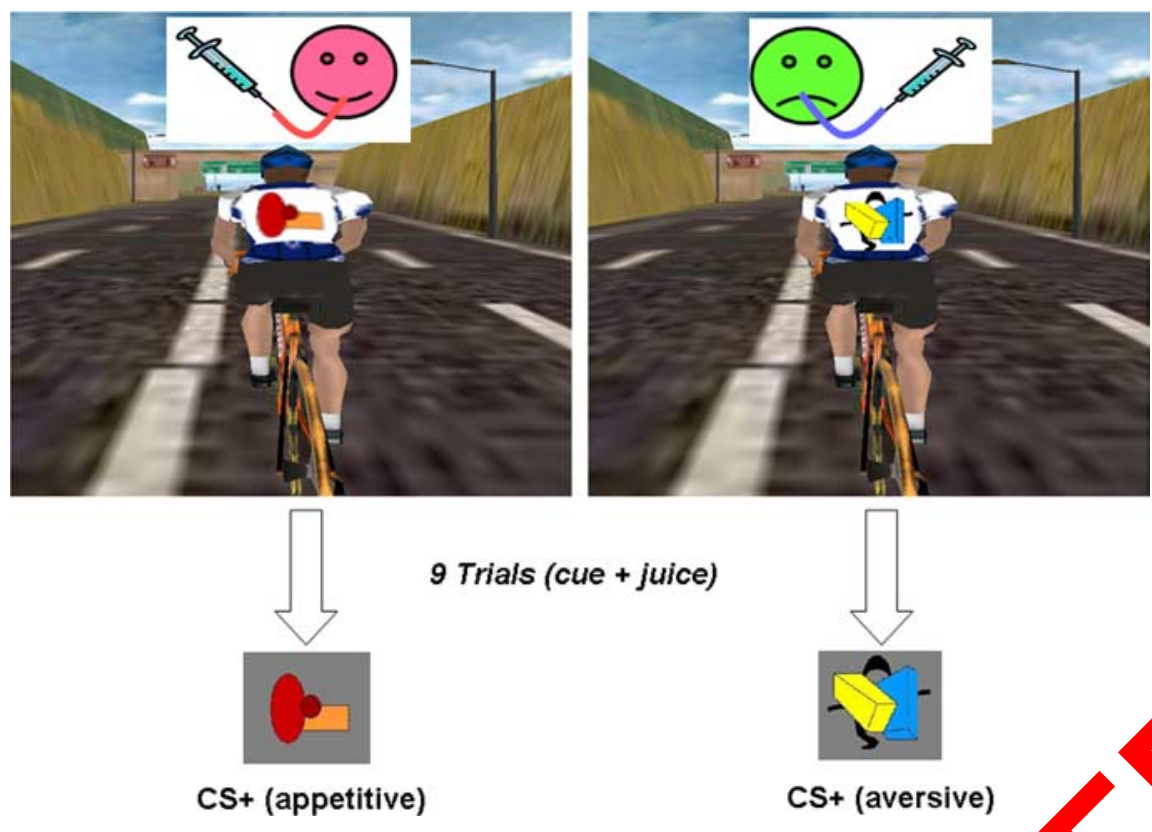

Figure 1. Day 1: Pavlovian conditioning in virtual environment. Participants in the virtual cycle race we competitors. The stimuli on the competitors' jerseys acted as CSs predicting the delivery of either pleasant or unpleasant juic stimulus-to-juice assignment was counterbalanced across participants.

ian conditioned stimulus for an individually specified appetitive drink (e.g., orange juice), the other for an individually specified aversive drink (e.g., salty tea). Subsequently, stimuli conditioned in the virtual world were presented in dentally in the real world (embroidered onto tow ${ }^{\prime}$ a wa ing room) and their effects on participants' chavi wer observed. Later, the CSs were presented as S es (d turextinction) in the magnetic resonance ima ng scanner and the brain responses to them were $o$ npared. $r$ analysis of functional MRI (fMRI) data is fo sed the inst. given that this region may play a cruci role in ta -outcome representations in animal conditi ng tasks (Cub o et al., 1999; Balleine and Dickinson, $20 \%$. Of re in humans is that the anterior insula is activated du nticip on visually presented foods (O'Dohe aver al., 2006) and, more generally, appears have role in pping primary, visceral sensory states to heir motivational properties (O’Doherty, 2004)

\section{Materials and Methods}

Twenty-two healthy participants (14 males; age range 18-44) gave written informed consent. The study was approved by the Local Research Ethics Committee.

All participants underwent pavlovian conditioning involving exposure to two abstract stimuli within a virtual environment (Fig. 1). One stimulus predicted aversive juice, the other appetitive juice. The impact of this conditioning was tested during acquisition and then, $72 \mathrm{~h}$ later, outside the virtual environment.

Conditioning phase: virtual environment. Participants made an initial selection of five favorite and five least favorite drinks. This was a partially constrained choice: they were asked to avoid choosing alcoholic, carbonated, or caffeinated drinks. The highest rated drink was presented (participants were given fixed $3 \mathrm{ml}$ delivery of each fluid via the experimental syringe pump mechanism) and was blindly rated on a 20 point Likert scale [+10 ("really like") to -10 ("really dislike")]. Only a drink with a rating of greater than +5 was considered suitable as the appetitive US.

For the aversive US, cold tea with a standard amount of salt was subject to this tasting. If ratings failed to reach -5 or less, more salt was added, and it was retested.

Participants, seated in front of a computer monitor, were told to imagine that they were taking part in a cycling race in a virtual town and that they were testing a new fluid replacement strategy using frequent, small juice deliveries. These fluids would be delivered by support cars and fellow cyclists and they must try to distinguish which cars/cyclists would give pleasant and which would give unpleasant juices. Thus, the bicycles and cars functioned as CS either followed by a juice (CS+) or, in the case of a subset of the cars, by no outcome (CS-). We focus on the relationship between $\mathrm{CS}+\mathrm{s}$ on the jerseys of the cyclists, and the drink d very each of these stimuli predicte We refer to hese as CS+app (stimulus predio the pleas $t$ juice) and CS $+a v$ (the imulus edicted le unpleasant juice).

Delivery drink $(0.9 \mathrm{ml})$ occurred by $\mathrm{PC}$. ontrolled automated syringe pumps sewEra) d custom $\mathrm{C}++$ software controlg $450 \mathrm{ml}$ syringes linked via Portex hygie tubing (800/010/225/800: Fischer Scientific).

The three-dimensional (3D) environment was developed in Microsoft Visual $\mathrm{C}++$ / ATL-COM using the DirectX 9.0c framework (icrosoft $\mathrm{C}$,poration) and a custom-written in-house Virtual Real engin "Virtual Environment"). Experiments were run on a Windows-based high-end Dell laptop with a $256 \mathrm{MB}$ GeForce graphard. The cyclist 3D animation model was purchased from the3 Dstudio.com and additional graphics, scenes and animations for the Virtual Environment were created in house. The animation model was converted to DirectX format. The abstract stimulus was created using Photoshop (Adobe) and served as the visual element of the cyclist that predicted juice delivery (the CS).

During conditioning, cyclists and cars drove past the player, appearing from behind on the left or right so that the participant had the experience of being overtaken. A single trial (competitor appears and accelerates into distance) lasted $4.5 \mathrm{~s}$. During the final second the fluid was delivered. Nine presentations of each CS were distributed across day 1 training.

Recording approach/avoidance behavior. Participants controlled their trajectory with a button box (Fig. 2). They were given prior instructions to chase ("approach") the stimuli that predicted pleasant juices and veer away from ("avoid") those predicting unpleasant juices. This avoidance/ approach behavior was recorded to assess learning but did not influence juice delivery. The default forward speed (at which participants traveled without the need for control) was four "screen units" per second (each screen unit corresponding to $\sim 6$ pixels. Thus, if participants did not respond to a CS, they would attain a particular end-position for each trial. This was set to zero so that all deviation from this position could be recorded as approach or avoidance behavior. For each trial the distance from this zero point was calculated. A positive value indicated that the participant had approached the CS. A negative value indicated avoidance.

Test phase 1: real-world incidental decision. Seventy-two hours later, the two conditioned stimuli were represented in the real world (Fig. 3). To minimize context overlap, this took place in a different building from prior conditioning. While the scanner was readied, participants were invited into a waiting room containing two chairs, each bearing a towel embroidered with one of the two CSs. The question here was whether participants would be influenced in their choice of seat depending on whether it bore CS+app or CS+av. Stimulus allocations to appetitive/ aversive conditions were fully counterbalanced among all participants ensuring that seat choice did not depend on stimulus allocation or chair position. 
The experimenter remained outside the room, entering after a couple of minutes to note which seat had been chosen.

Test phase 2: fMRI. All 22 subjects underwent fMRI which included a series of conditioning manipulations with the other stimuli (cars) to which they had been pre-exposed. The first scanning task focused on the CS $+\mathrm{s}$ which had appeared on the seats in the waiting room. These were presented intermixed with other car CSs and "filler" items (Fig. 4). Each stimulus was presented a total of seven times with a mean ITI of $3 \mathrm{~s}$ (SOA $4.5 \mathrm{~s}$ ). For technical reasons, the data on the CS + were available on a subset of 13 participants. The imaging analysis was limited to this subset, seven of whom had chosen the seat bearing the CS+app and six the CS+av. The subset was matched with the rest of the group in terms of gender and age and, within the subset, participants who had chosen the $\mathrm{CS}=$ app chair were representative of the other nine participants who were not scanned (all of whom had chosen the CS + app chair) in terms of personality and VR immersion scores (both measures having been taken on day 1 ). We used a Siemens Trio scanner operating at 3 Tesla. 674 gradient echo $\mathrm{T} 2^{\star}$-weighted echo planar images depicting blood oxygenation level dependent contrast were acquired.

Data were analyzed using statistical parametric mapping in the SPM5 program (www. fil.ion.ucl.ac.uk) including spatial realignment, normalization, smoothing $(6 \mathrm{~mm}$ fullwidth at half-maximum) and high pass filtering (cutoff 1/120 Hz). Events were modeled using a canonical hemodynamic response function (plus first derivative) convolved with a $\delta$ function placed at the onset of stim ulus presentation. The responses to CS + app and CS + av were modeled com red with baseline and contrast images t ${ }^{2} \mathrm{n}$ forward to a group analysis treating i ersubject variability as a random effect the $g$ ap level a $2 \times 2$ factorial ANOV no 1 was implemented to identify eura spons distinguishing the ap ctitive and a r e stimuli and determi wheth this wound be different depending o ve searcme. We focused on the area of as rior insula known to differentiate disgusting om appetizing foods in a sphere of interes (radius $=10$ $\mathrm{mm}$ ) centered on coordinates from a previous study $(x / y / z=36 / 26 /-6)$ (Beaver et al., 2006). A subsequent exploration of ventral striatum and a whole brain analysis did not identify activations surviving an appropriate correction for multiple comparisons.

\section{Results}

\section{Conditioning: day 1}

All participants rapidly acquired appropriate approach/avoidance behavior to CSs (Fig. 2), indicating learning within the virtual environment. Importantly, the learning in those subjects who, in the subsequent phase of the experiment, $72 \mathrm{~h}$ later, did not show evidence of transfer across

\section{Day 1 (virtual environment)}

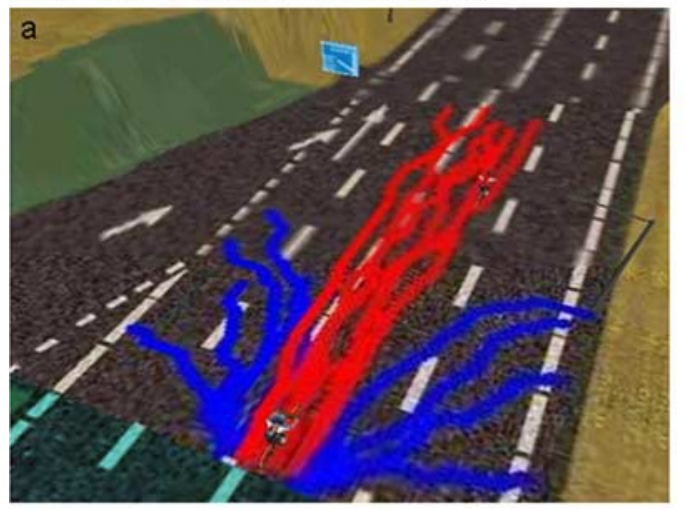

- -Typical approach behavior

- Typical avoidance behavior

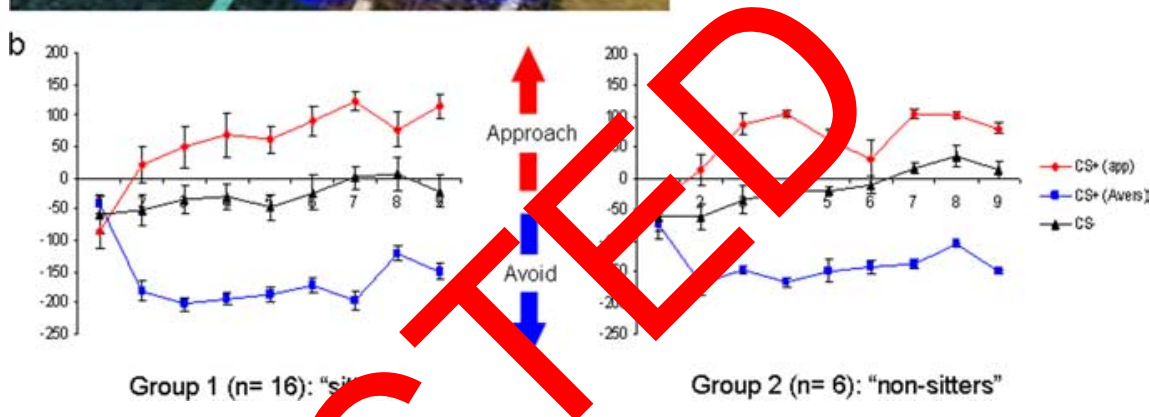

Figure 2. Day 1: Approach a avoidance be vior. After conditioning across several trials, participants learned to approach or avoid competitors $a$ ending on th bstract stimulus on their jerseys. $\boldsymbol{a}$, The typical trajectories are represented in blue ("avoid") and red (" $a$. roach") f a representative participant. $\boldsymbol{b}$, Group behavior for (S + app and (S + av

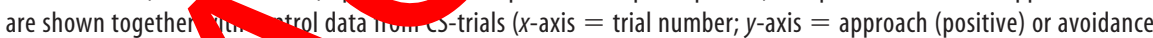
(negative) behavior). e exp those who when offe the eal-world choice three days later, chose to sit on a chair emblazoned with the CS+app. "Non- cers". pse who pose the chair with CS+av.

\section{Da) (rearworld)}

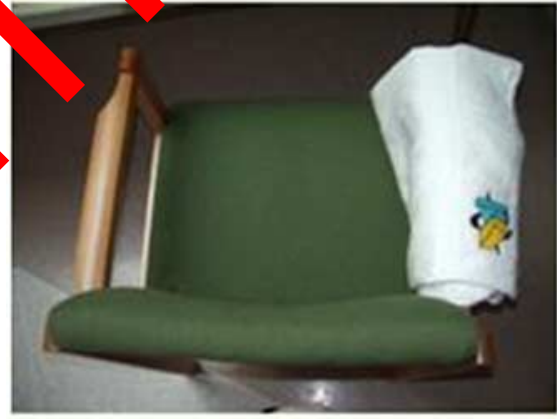

$27 \%$ - aversive
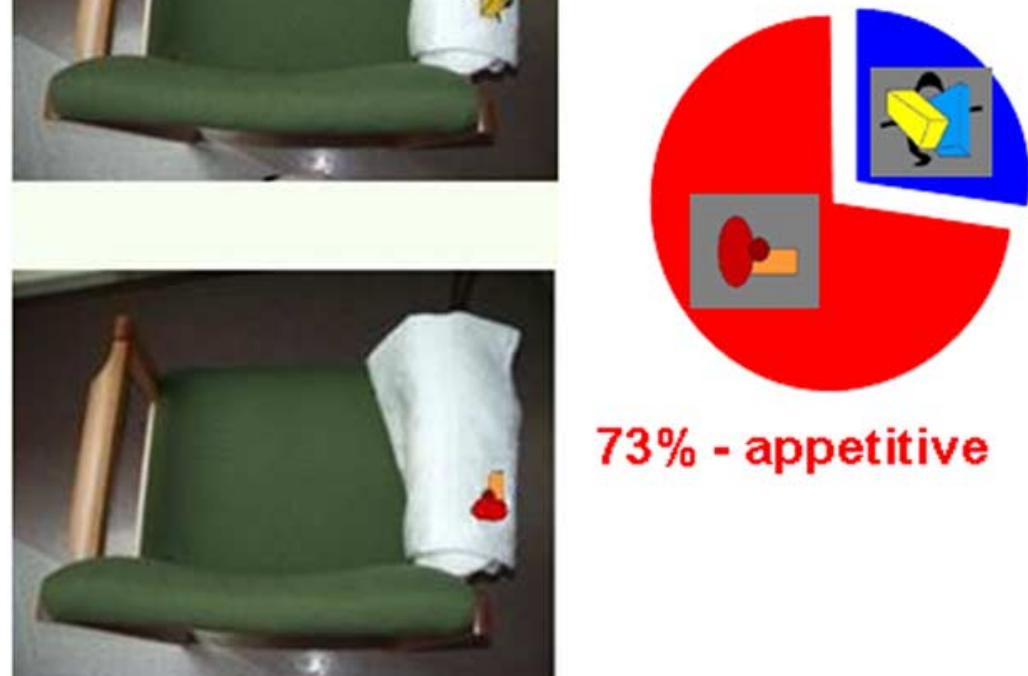

$73 \%$ - appetitive

Figure 3. Day 4: Real-world decision when asked to take a seat in an unoccupied waiting room before scanning. Sixteen participants chose the seat bearing a towel with the $C S+$ app. 


\section{Day 4 - Two-dimensional slideshow in scanner}
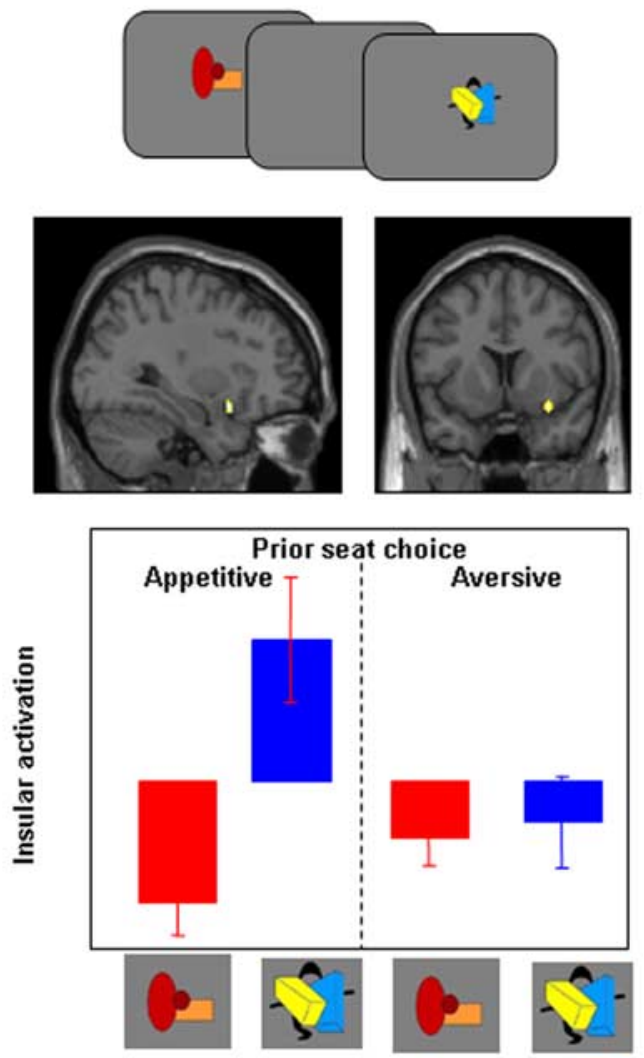

Stimulus presented

Figure 4. Day 4: Brain responses to $C S+s$. Stimuli conditioned in Figure 4. Day 4: Brain responses to $\mathrm{CS}+\mathrm{s}$. Stimuli conditioned in tirtual e ironmen
were shown in the scanner. Participants viewed each stimulus passic for $1.5 \mathrm{~s}$ stimulus interaction in the insula is shown. Here, differential act cion to ppand $C 5+a v$ was found only in participants who sat on the chair bearing appetitive aeak at 34/16/12; small volume corrected in $10 \mathrm{~mm}$ sphere around p rou of aversiv a eliciting stronger activation than appetitive food:36/26/-6; Reaver et al., $2 u$ The $y$-axis on the graph shows the parameter estimates (arbitrary units) $x$-axis shows the fferent CSs (following the schemes of Figs. 1,3). The red stimulus resents th $\mathrm{s}+$ app and the blue/yellow represents $C S+$ av.

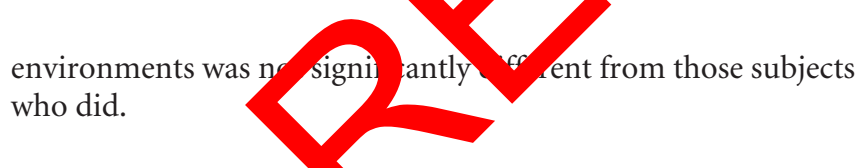

Seat choice: day 4

Sixteen participants $(73 \%$ chose the seat bearing the towel embroidered with the CS + app (Fig. 3) ( $\left.\chi^{2} 4.54 ; p<0.05\right)$. All denied deliberately choosing a seat on the basis of the stimuli. These 16 participants differed from those who chose the chair with the CS + av in that they showed lower impulsivity scores (7.4 \pm 1.8 and $10.1 \pm 2.3$, respectively, as measured on the BAS drive scale (Carver and White, 1994) $p<0.01$ ) and a trend $(21.6 \pm 3.9$ and $17.8 \pm 5.1$ respectively, $p=0.074)$ toward greater levels of subjective immersion (Schubert et al., 2001) in the environment during their prior exposure. The groups did not differ in their previous experience or frequency of computer game playing.

Brain responses to CS: day 4

There were marked differences between participants in their insula responses to the stimuli (Fig. 4) depending on their prior seat choice. Activity in this region distinguished between CS+app and CS + av in the group who chose the chair bearing the CS + app but not in the minority of participants who chose the other chair (group-by-stimulus interaction $(p=0.015$ with small volume correction). This was seen in the ventral ROI but was also apparent dorsally and bilaterally at an uncorrected threshold $(p<$ 0.01; see supplemental Table, available at www.jneurosci.org).

\section{Discussion}

Our data show that pavlovian conditioning can transfer from the virtual world to an incidental choice in the real world. The CSs carried their motivational properties across the different environments, although participants showed no evidence of being aware that this was so. Moreover, the impact on the precise nature of conditioning (aversive versus appetitive taste) was primarily seen in insular cortex but this was significantly attenuated in the participants who did not show transfer. This subgroup w? distinct in personality scores and in their sense of immersi in the ronment.

Much concern has been ressed ove he impact of widespread use of virtual environn $A$ ts an comput games (Block, 2008). A counter-argument $t$ such fears th earning and motivational states operate exc vively hin the histly constrained context of a "fantasy" clear" dif cated fr $A$ the real world. Indeed, a recent UK Advert 'g Standa Au ority ruling (http://www.asa.org.uk/ asa/adju_cat S/Public/1__ADJ_43426.htm) rests on this distinction. Our finding however, show that objects can carry their moti$\mathrm{y}$ - ona attributes $\mathrm{b}$ ond the virtual world.

Although it is known that animals approach appetitive CSs Hearst and enkins, 1974), to the best of our knowledge the p. cent sty $y$ is the first to show that such CSs control comparas chavior in humans. Humans are faster to perform a -onse that makes a positively-valenced word approach rather than recede (Solarz, 1960), a tendency that is modulated by hunger in the case of food-related words (Staats and Warren, 1974). Moreover, pavlovian-instrumental transfer procedures demonstrate that an appetitive CS augments human performance of an instrumental response (Hogarth et al., 2007; Bray et al., 2008). Our finding is congruent with these demonstrations that an appetitive CS has a significant impact on human behavior. Complementary to lesion work [for review, see Balleine and Ostlund (2007)], fMRI findings from purely appetitive conditioning have suggested the importance of amygdala and striatum in transfer (Bray and O'Doherty, 2007; Talmi et al., 2008). Our use of aversive conditioning as well as the incidental nature of the transfer test may explain the absence of striatal effects (at corrected thresholds) here.

In the current study, the striking finding in the brain was the differentiation of insula activation between aversive and appetitive stimuli in participants who transferred learning from the virtual to the real world but not in those who did not. These findings concur with, and extend, the notion that the insula is involved in assigning motivational value to conditioned stimuli (Balleine and Dickinson, 2000), particularly with respect to aversive food conditioning (Beaver et al., 2006). Interestingly, the anterior insula has been proposed to play a role in linking prospective aversive body states with avoidance behavior (Paulus and Stein, 2006). Perhaps activity in response to the CS + av led to avoidance of the chair bearing this stimulus in those who showed this transfer but not those in whom it was not apparent.

While we know that basic associative conditioning phenomena can occur within virtual worlds (Hamilton and Sutherland, 1999; Grillon et al., 2006; Alvarez et al., 2007; Doeller and Burgess, 2008), the current observation is notable for the fact that the CSs maintained their motivational attributes, for the majority of the participants, across two environments that differed so greatly. 
Exposure to the virtual environment took place in a different setting, indeed in a different building, three days before testing the residual motivational characteristics of the CSs. The fact that CSs retained motivational properties is particularly noteworthy given that reexposure to a CS alone may be insufficient to activate a cue incentive memory. Moreover, extinction of a CS-US relationship in one part of a virtual environment may not extend to another part of that environment (Alvarez et al., 2007). It is possible that context, acting as an "occasion setter" (Holland, 1985; Bouton and Swartzentruber, 1986), or perhaps overlapping task demands (Lee and Everitt, 2008) could contribute to retained action of CSs. In our study, overlap of context could have arisen through the presence of the same experimenters, although they were not present during seat choice. This possibility would require further study and raises the additional question of whether the minority of participants who appeared insensitive to the CS (that is, those subjects who sat in the "aversive" seat) were actually more sensitive to the context change than the majority. Of central interest, however, and of relevance to the impact of VR on real-world behavior, is that CSs maintained their motivational properties, even when the environment and the task were radically changed. We can only speculate on how this impact could grow as the environments in which computer games are played begin to overlap more and more with the real world, particularly given the trend of setting games within remarkable facsimiles of real places.

There was reduced evidence of the transfer effect in participants with higher impulsivity scores. This was associated with the absence of a distinction between CS + av and CS + app in insular cortex in this subgroup. One possibility is that the CS+av dic in these participants, create the inhibitory drive that might lea to its avoidance. This is, however, a highly speculative idea, som what undermined by the fact that avoidance beha ors a mon traditionally thought to be measured by the BIS ale. Ul mately, while the reported correlation is interesting its tion of the relationship requires further ady. W regard to the trend toward different VR immer on cores acro the two subgroups, this does not equate th how eng ing participants found the task but rather with ow "realistic hey found the experience of the virtual eny nmen $n$ which they played the game.

Our observations sh- - be ta n into count amid the current, general discuss in col ernins effects, harmful or otherwise, of increasi comp winespread use of games (in the UK estimated that $51.2 \%$ of males aged 10-35 play such games, here has been much debate surrounding their possibly harmful huences. As well as having a clear and direct influence on reward systems in the brain (Koepp et al., 1998), and general effects on individuals' levels of aggression (Anderson et al., 2007), it has been proposed that internet usage produces effects comparable with those seen in substance addiction (Block, 2008), although this is disputed (Wood, 2008). Our findings suggest that we should be mindful not simply of how the individual responds at a general level but also of the effects that such games may have upon the real-world motivational characteristics with which particular stimuli become imbued as a consequence of conditioning within the game environment. This may be especially important given increasing levels of exposure. One could argue that our use of "real" appetitive and aversive stimuli (juices) is in contrast to the virtual outcomes (e.g., points) available in computer games, rendering it difficult to generalize the findings in this way. However, we recognize that rewards and punishments within computer games are becoming ever more vivid and realistic. For this reason, we suggest that this stimulus- specific conditioning should be taken seriously when considering the etiology of online and gaming related behaviors and in predicting the consequences, both harmful and beneficial, of the virtual world on real-world behavior and choice.

\section{References}

Alvarez RP, Johnson L, Grillon C (2007) Contextual-specificity of shortdelay extinction in humans: renewal of fear-potentiated startle in a virtual environment. Learning and Memory 14:247-253.

Anderson CA, Gentile DA, and Buckley KE (2007) Violent video game effects on children and adolescents: theory, research and public policy. Oxford: Oxford UP.

Balleine BW, Dickinson A (2000) The effect of lesions of the insular cortex on instrumental conditioning: evidence for a role in incentive memory. J Neurosci 20:8954-8964.

Balleine BW, Ostlund SB (2007) choice point: action selection and initiation in instrum tal condit ing. Ann NY Acad Sci 1104:147-171.

Beaver JD, Lawrence AD A Ditzh zen J, D s MH, Woods A, Calder AJ (2006) Individual cerences in re dre predict neural responses to images of food.

Block JJ (2008) ssue DSM-V ternet addiction. Am J Psychiatry 165:306

Bouton (1986) Analysis of the associative and occasion-sett properties of contexts participating in a Pavlovian dis'nation. J Ex Psychol Anim Behav Process 12:333-350. y S, O'Doherty J (2v07) Neural coding of reward-prediction error signals durir classical conditioning with attractive faces. J Neurophysiol 97:3036-3 5

B S, Rano A, Shimojo S, Balleine B, O’Doherty JP (2008) The neural mus underlying the influence of pavlovian cues on human deci-

cion making. J Neurosci 28:5861-5866.

Carin AS, Hoffman HG, Weghorst S (1997) Virtual reality and tactile augmentation in the treatment of spider phobia: a case report. Behav Res Ther 35:153-158.

Carver CS, White TL (1994) Behavioral inhibition, behavioral activation, and affective responses to impending reward and punishment: the BIS/ BAS scales. J Pers Soc Psychol 67:319-333.

Cubero I, Thiele TE, Bernstein IL (1999) Insular cortex lesions and taste aversion learning: effects of conditioning method and timing of lesion. Brain Res 839:323-330.

Doeller CF, Burgess N (2008) Distinct error-correcting and incidental learning of location relative to landmarks and boundaries. Proc Natl Acad Sci U S A 105:5909-5914.

Emmelkamp PM, Krijn M, Hulsbosch AM, de Vries S, Schuemie MJ, van der Mast CA (2002) Virtual reality treatment versus exposure in vivo: a comparative evaluation in acrophobia. Behaviour Research and Therapy 40:509-516.

Grillon C, Baas JM, Cornwell B, Johnson L (2006) Context conditioning and behavioral avoidance in a virtual reality environment: effect of predictability. Biol Psych 60:752-759.

Hamilton DA, Sutherland RJ (1999) Blocking in human place learning: evidence from virtual navigation. Psychobiology 27:453-461.

Hearst E, Jenkins HM (1974) Sign tracking: the stimulus-reinforcer relation and directed action. Austin, TX: Monograph of the Psychonomic Society.

Hogarth L, Dickinson A, Wright A, Kouvaraki M, Duka T (2007) The role of drug expectancy in the control of human drug seeking. J Exp Psychol Anim Behav Process 33:484-496.

Holland, PC (1985) The nature of conditioned inhibition in serial and simultaneous feature negative discriminations. In: Information processing in animals: conditioned inhibition (Miller RR, Spear NE, eds), pp. 267297. Hillsdale, NJ: Erlbaum

Koepp MJ, Gunn RN, Lawrence AD, Cunningham VJ, Dagher A, Jones T, Brooks DJ, Bench CJ, Grasby PM (1998) Evidence for striatal dopamine release during a video game. Nature 393:266-268.

Krakauer JW (2006) Motor learning: its relevance to stroke recovery and neurorehabilitation. Curr Opin Neurobiol 19:84-90.

Lee JL, Everitt BJ (2008) Reactivation-dependent amnesia for appetitive 
memories is determined by the contingency of stimulus presentation. Eur J Neurosci 15:390-393.

O'Doherty JP (2004) Reward representations and reward-related learning in the human brain: insights from neuroimaging. Curr Opin Neurobiol 14:769-776.

Park J, MacRae H, Mussleman LJ, Rossos P, Hamstra SJ, Wolman S, Reznick RK (2007) Randomized controlled trial of virtual reality simulator training: transfer to live patients. Am J Surg 194:205-211.

Paulus MP, Stein MB (2006) An insular view of anxiety. Biol Psych 60:383-387.

Pull CB (2008) Recent trends in the study of specific phobias. Current Opinion in Psychiatry 21:43-50.

Rothbaum BO, Hodges LF, Ready D, Graap K, Alarcon RD (2001) Virtual reality exposure therapy for Vietnam veterans with posttraumatic stress disorder. J Clin Psych 62:617-622.

Schubert T, Friedmann F, Regenbrecht H (2001) The experience of presence: factor analytic insights. Pres Tele Virt Envir 10:266-281.

Solarz AK (1960) Latency of instrumental responses as a function of compatibility with the meaning of the eliciting verbal signs. J Exp Psychol 59:239-245.

Staats AW, Warren DR (1974) Motivation and three-function learning: food deprivation and approach-avoidance to food words. J Exp Psychol 1974:1191-1199.

Talmi D, Seymour B, Dayan P, Dolan RJ (2008) Human pavlovianinstrumental transfer. J Neurosci 28:360-368.

Wood R (2008) Problem with the concept of video game "addiction": some case study examples. Int J Ment Health Add 6:169-178.

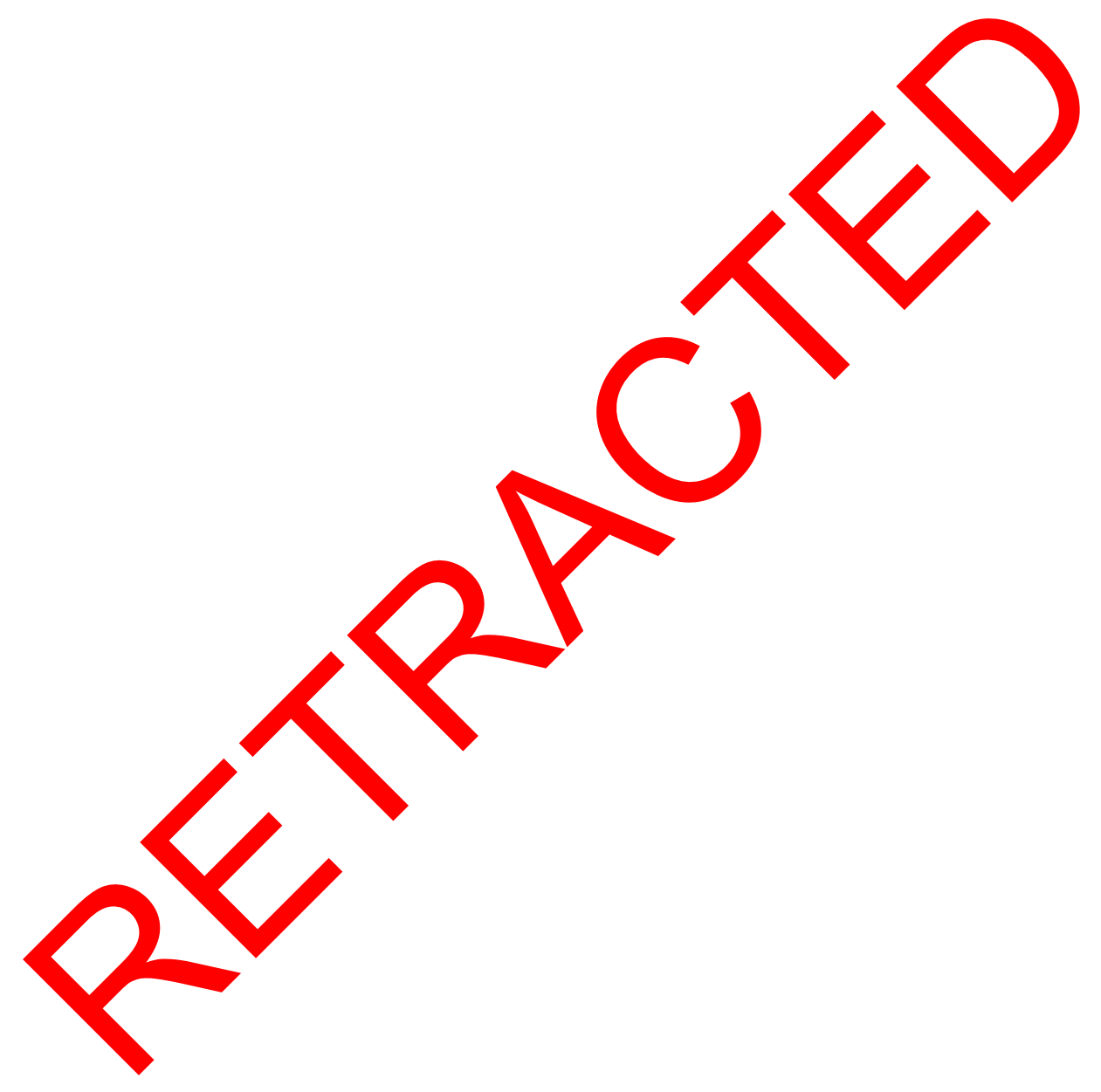

